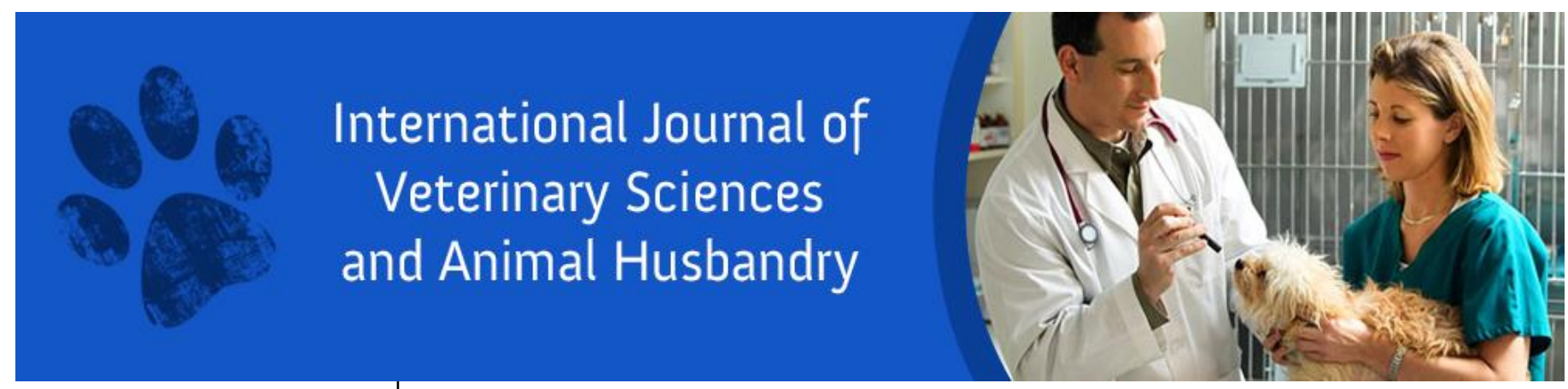

ISSN: $2456-2912$

VET 2021; 6(2): 28-30

(C) $2021 \mathrm{VET}$

www.veterinarypaper.com

Received: 19-01-2021

Accepted: 21-02-2021

Deshpande Smita Sharadrao

Post Graduate Scholar, Department

of Veterinary Microbiology, College of

Veterinary Sciences and Animal

Husbandry, Maharashtra Animal and

Fishery Sciences University, Nagpur,

Maharashtra, India

\section{Deshpande Anand}

Professor and Scientist Department of

Veterinary Microbiology, College of

Veterinary Sciences and Animal

Husbandry, Maharashtra Animal and

Fishery Sciences University, Nagpur,

Maharashtra, India

\section{Suryawanshi Prashant}

Assistant Professor Department of

Veterinary Microbiology, College of

Veterinary Sciences and Animal

Husbandry, Maharashtra Animal and

Fishery Sciences University, Nagpur,

Maharashtra, India

Gaikwad Satish

Assistant Professor Department of

Veterinary Biotechnology, College of

Veterinary Sciences and Animal

Husbandry, Maharashtra Animal and

Fishery Sciences University, Nagpur,

Maharashtra, India

\section{Manjarmkar Kshitija}

Post Graduate Scholar, Department

of Veterinary Microbiology, College of

Veterinary Sciences and Animal

Husbandry, Maharashtra Animal and

Fishery Sciences University, Nagpur,

Maharashtra, India

Pathan Nazia

Post Graduate Scholar, Department of Veterinary Microbiology, College of Veterinary Sciences and Animal

Husbandry, Maharashtra Animal and Fishery Sciences University, Nagpur, Maharashtra, India

\section{Rising antimicrobial resistance pattern among the bacteria isolated from wound cases of domesticated animal in India}

\author{
Deshpande Smita Sharadrao, Deshpande Anand, Suryawanshi Prashant, \\ Gaikwad Satish, Manjarmkar Kshitija and Pathan Nazia
}

DOI: https://doi.org/10.22271/veterinary.2021.v6.i2a.332

\section{Abstract}

Antimicrobial resistance gained by the microorganisms is a global threat, which leads to economic loss as there will be treatment failure, loss of life, discovery of newer and better antimicrobials of any infections as there is need for discovery of newer and better antimicrobials. A total of forty five samples were collected from clinical wound cases in and around Parbhani Veterinary Hospitals. The total of 39 Gram negative and 17 Gram positive isolates includes Pseudomonas (24), E. coli (15) and Staphylococcus (17) spp, were confirmed by biochemical characterization. Susceptibility of isolates to seven antibiotics was tested using disc diffusion method according to the guidelines defined by Clinical Laboratory Standard Institute. Pseudomonas Isolates showing resistance to antibiotic for Ceftazidime, Levofloxacin were for E.coli isolates showing resistance to antibiotics Erythromycin, Tetracycline and For Staphylococcus isolates Penicillin and Enrofloxacin were resistance.

Keywords: animal, wound, bacteria and antimicrobial sensitivity

\section{Introduction}

Wound is defined as the loss or breaking of cell and anatomic or functional continuity of living tissues which might not handiest be prompted by using the external factors, but also as a hardship of a few disorder processes and different internal factors (Ayello, 2005) ${ }^{[2]}$. Wounds are common and may be challenging to manipulate in each human and animals, specifically non-recuperation wounds and wounds with secondary infection by using multidrug resistant bacteria. (Atiyeh et al., 2009) ${ }^{[1]}$. Wound therapies have evolved significantly over the past three decades and provided more effective and easily healing methods such as dressings, wound bed preparations, quaterization and ligation. Technology and product proliferation, however, varied by geography, usually as a function of economics and health care policy. A number of research conducted recently discovered that for septic wounds, the most common isolates were Staphylococcus aureus, Pseudomonas aeruginosa, Escherichia coli, Klebsiella spp. and Acinetobacter spp. The management of wound infections has grown to be difficult because of the great bacterial resistance to antibiotics and extra incidence of infections because of poly-microbial plant life (Pallavali et al., 2017) ${ }^{[8]}$. The use of antibiotics in the treatment of bacterial infections increasingly encounters difficulties caused by the emergence and rapid spread of pathogenic bacteria exhibiting multidrug resistance.

\section{Materials and Method}

\subsection{Sample collection}

Forty five wound swab and pus samples were collected in sterile vials for collection of samples from various injured animals. The samples were marked with position, date of collection and age of the animal. The sampling area includes local dairies, animal farms and state veterinary clinics. The samples were transported on ice to the laboratory and stored at 4 ${ }^{\circ} \mathrm{C}$ until processing. The samples were collected from March 2018 to April 2019. 


\subsection{Bacterial examination and confirmation}

The cultural characters of the typical colonies were studied. The smears were crafted from various colonies and stained by means of Gram's staining method. Hanging drop methods were used to determine the motility of organisms. Primarily, organisms were recognized by their morphological, cultural and staining characters and confirmation was made by method explained by Cruickshank et al., (1975) ${ }^{[4]}$

\subsection{Antimicrobial susceptibility test 2.3.1 Agar diffusion method}

All the bacterial isolates were subjected to antibiotic sensitivity test using standard antibiotic discs by the method

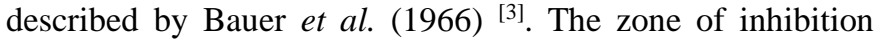
(diameter in $\mathrm{mm}$ ) of each antibiotic disc was then correlated with sensitivity as per standard chart supplied along with disc and results recorded in terms of either sensitive or resistant bacteria to the respective antibiotics.

\section{Results}

In the present study a total of 45 infected wound cases in which 11- cattle,7- goat, 8- Buffalo, 5- Horse, 14- Dog samples were included in this study. Monomicrobial infection was present in 15 cases, whereas mixed infection by two bacteria was observed in 30 cases. Gram negative bacteria were isolated frequently [30/45 (52.8\%)] than gram positive bacteria. The most common Gram-negative isolates were
Pseudomonas aeruginosa (24). Dhawan et al., (2006) [5] reported similar findings though it was $5.63 \%$ isolated from wound cases were Escherichia coli (15) and Pseudomonas putida (02). Among Gram-positive bacteria that is Coagulase positive Staphylococcus aureus (5) and Coagulase negative staphylococcus (8) and Enterococcus faecalis (4) were observed (Table 1). Acharya et al., (2008) in their studies conducted in Nepal reported Gram positive cocci constituted $57.06 \%$ and Gram negative bacteria constituted $42.94 \%$ isolates. In this study the bacterial pathogens of wound were isolated and it was found that, among major isolates Pseudomonas aeruginosa was the most common isolate (37.3\%). This finding is in contrast with the findings of Tiwari et al., (2016) ${ }^{[9]}$ who reported Staphylococcus aureus as major bacteria isolated from the wound cases and The bacteria which were identified are presented in Table 2.

Table 1: Bacteria isolated from clinical wound following cases

\begin{tabular}{|c|c|}
\hline Bacteria isolated $(\mathbf{n = 5 6})$ & Number \\
\hline Gram-negative bacteria $(\mathbf{n}=\mathbf{3 9})$ & \\
\hline Pseudomonas aeruginosa & 22 \\
\hline Pseudomonas putida & 02 \\
\hline Escherichia coli & 15 \\
\hline Gram-positive bacteria $(\mathbf{n}=\mathbf{1 7})$ & \\
\hline Staphylococcus aureus & 5 \\
\hline Coagulase negative staphylococcus & 8 \\
\hline Enterococcus faecalis & 4 \\
\hline
\end{tabular}

Table 2: Morphological and cultural characteristics of bacterial isolates

\begin{tabular}{|c|c|c|c|c|}
\hline Sr. No. & Pathogen & Media used & Colony characteristics & Gram stain and morphology \\
\hline 1. & Pseudomonas aeruginosa & Cetrimide agar & Yellow green, fluorescent & Negative (Rod) \\
\hline 2. & Escherichia coli & EMB agar & Metallic sheen coloured & Negative (Rod) \\
\hline 3. & Enterococcus faecalis & Nutrient agar & Smooth, cream/white & Positive (Cocci) \\
\hline 4. & Staphylococcus aureus & Mannitol salt agar & Smooth, Golden yellow & Positive (Cocci) \\
\hline
\end{tabular}

The 37 Gram negative bacteria and 17 Gram positive bacteria isolates from clinical wound cases were grown on discriminatory media and further subjected to biochemical characterization by MR, VP, Indole and Nitrate test. The suspected isolates of both species were subjected to different sugar fermentation tests. For Pseudomonas spp. different sugars viz. Glucose, Lactose, Arabinose, Rhamnose, Mannitol, Dulcitol, Inositol, and Sucrose were used. For assumed Staphylococcus spp different sugars viz. Glucose, Rhamnose, Maltose, Arabinose, Mannitol, Dulcitol, Xylose, Raffinose, Inositol, Sucrose, Lactose were used. The clinical wound isolated bacteria of Pseudomonas spp. were subjected to different tests such as Catalase, Citrate, Urease, and Haemolysis. Similarly Catalase, Citrate, Coagulase, Urease and Haemolysis were done for detection of suspected characters of Staphylococcus spp. The results of biochemical tests for Pseudomonas spp were MR,VP and Indole tests negative whereas Nitrate test was positive exceptional for $P$. putida spp. For sugar fermentation test all positive Pseudomonas spp fermented glucose, Arabinose but did not fermented to Inositol, Lactose, Dulcitol and Sucrose. The results of different tests for detection of different characters of Pseudomonas spp are that all 24 Pseudomonas spp were positive for Catalase, Urease and Citrate where as hemolysis test negative. Similar work is done by Mokate and More (2013) who have reported the isolation and identification of Pseudomonas spp by using biochemical tests.

\subsection{Antimicrobial susceptibility of Pseudomonas spp, E. coli and Staphylococcus spp.}

Antimicrobial susceptibility test was performed on 45 bacterial spp. by agar disc diffusion method and as many as 7 antibiotics for Pseudomonas, 7 antibiotics for E.coli and 7 antibiotics for Staphylococcus spp. were used for studying the resistance and sensitivity pattern of Bacterial species. Among the $P$. aeruginosa isolated, all the antibacterial antibiotics were most lively with $100 \%$ susceptibility to Gentamicin followed by Ciprofloxacin (80\%), Ceftazidime (28.5), Meropenem (54.7\%), Imipenem (38\%), Levofloxacin (28\%) and Amoxicillin (57.7\%). For E. coli isolates were $100 \%$ sensitive to Gentamicin, followed by Chloramphenicol (95\%), Ceftriaxone (85\%), Norfloxocin (20\%), Erythromycin (37\%), Ciprofloxacin (57\%), and Tetracycline (28\%). and for Staphylococcus isolates were $100 \%$ sensitive for ciprofloxacin followed by Chloramphenicol (87\%), Gentamicin (67\%), Amoxicillin (37\%), Penicillin (5\%), Enrofloxacin (10\%), Ampicillin (28\%).

\section{Discussion}

In the present study the wound infection is the most dynamic that retards the normal wound healing. Wounds are caused by various reasons such as trauma, injury during surgery etc. A wound is either simple with superficial injury or complicated with involvement of deeper structures e.g., muscle, tendons, vessels, bones etc. Infected wounds illustrate deferred epithelial growth and migration, superior cellular necrosis and 
lysis of collagen tissue. Bacteria are proficient of producing collagenase enzyme which degrades collagen tissue synthesized by fibroblast to decrease the tensile strength, and ultimately to case wound disruption. Wounds are congregation by a polymicrobial flora comprising synergistic bacteria, such as Pseudomonas, Staphylococci and Escherichia coli spp bacteria.

The injudicious uses of antibiotics throw in to the development of super infection and resistant microbial species. When a wound is treated with antimicrobial drug, a selective pressure is applied to all bacteria exposed to the drug. Pallavali et al., (2017) ${ }^{[8]}$ who demonstrated for Pseudomonas spp a relatively higher sensitive to gentamicin, Cefotaxime and the antibiotic resistance pattern demonstrated to ampicillin (80.7\%), amoxicillin (88.4\%). The sensitivity patterns exhibited by $E$. coli had been highly decrease than the ones in previous reports, which demonstrated ampicillin (96.6\%), tetracycline (79\%) and gentamicin (51.7\%)) a particularly higher percent of drug resistance than our findings within the current examine as quoted by Mama et al., (2014) ${ }^{[6]}$

The study conducted in Andhra Pradesh verified a relatively greater percentage of multi-drug resistance in comparison to our observations (95.5 to $100 \%$ ) S. aureus was $100 \%$ resistant to benzyl penicillin, ampicillin and had variable resistances to other tested drugs, including kanamycin (13.6\%), vancomycin (9\%), cefotaxime $(9 \%)$ and tobramycin $(4.7 \%)$ as reported by Pallavali et al., (2017) ${ }^{[8]}$.

\section{Conclusion}

It was observed that from clinical wound cases Pseudomonas (60\%), Staphylococcus $(30 \%)$ and E. coli $(10 \%)$ was present. The level of multidrug resistance to the commonly used antibiotics in veterinary hospitals has increased. In the present study Pseudomonas spp were resistant to antibiotics such as Ceftazidime (78\%), Imipenem (88\%) and Levofloxacin (90\%). Staphylococcus showed resistance to antibiotics viz. Penicillin (95\%), Enrofloxacin (90\%), Ampicillin (78\%). and E. coli were resistant to antibiotics Tetracycline (80\%) and Erythromycin $(88 \%)$. The antibiotic Gentamicin was observed to express sensitivity against Pseudomonas, Staphylococcus and $E$. coli organisms isolated from wound

\section{Acknowledgement}

This study was supported by Faculty of Veterinary Microbiology, Department of Veterinary Microbiology, Parbhani (MAFSU, Nagpur). The authors would like to thank the staff of COVAS, Parbhani for their guidelines as well as my family for their support during research work.

\section{References}

1. Atiyeh BS, Dibo SA, Hayek SN. Wound cleansing, topical antiseptics and wound healing. Journal of Inter Wound 2009;6(6):420-30.

2. Ayello EA. What does the wound say? Why determining etiology is essential for appropriate wound care. Journal of Advances Skin Wound Care 2005;18(2):98-109.

3. Bauer AW, Kirby WM., Sheris JC, Truck M. Antibiotic susceptibility testing by a standardized single disc method. Journal of Am. J Clin. Path 1966;145:225-230.

4. Cruickshank R, Duguid JP, Marmion BP, Swain RHA. Medical Microbiology. 12 $2^{\text {th }}$ Edition, Vol. II Churchill Livingstone, Edinburgh, London and New York $1975 ; 2 ; 1767-183$.
5. Dhawan B, Gadepalli R, Sreenivas V, Kapil A, Ammini AC, Chaudhry RA. A clinico-microbilogical study of diabetic foot ulcers in an Indian tertiary care hospital. Journal of Diabetes care 2006;29:1727-32.

6. Mama M, Abdissa A, Sewunet T. Antimicrobial susceptibility pattern of bacterial isolates from wound infection and their sensitivity to alternative topical agents at Jimma University Specialized Hospital, South-West Ethiopia. Annals of clinical microbiology and antimicrobials 2014;13:1-7.

7. Mokate AA, More SM. Isolation, Identification and Characterization of Pseudomonas species from Lonar lake for Production of Lipase. Journal of Int. J Curr. Microbiol. App. Sci. 2013;2(11):36-42.

8. Pallavali RR, Degati VL, Lomada D, Reddy MC. Isolation and in vitro evaluation of bacteriophages against MDR-bacterial isolates from Septic Wound infections. Journal of PLOS ONE 2017;12(7):0179245.

9. Tiwari R, Yadav SK, Singh S. Methicillin Resistant Staphylococcus aureus Isolated from Wounds of Livestock and Companion Animals of Uttar Pradesh India: A Preliminary Study. Journal of Int. J Pharmacol 2016;12(8):821-829. 